\title{
Rising Incidence of Acute Hospital Admissions due to Gout
}

\author{
Mark D. Russell ${ }^{1}$, Mark Yates, Katie Bechman, Andrew I. Rutherford, Sujith Subesinghe, \\ Peter Lanyon, and James B. Galloway
}

\begin{abstract}
Objective. To describe trends in acute hospital admissions due to gout in England, with rheumatoid arthritis (RA) as a comparator, alongside prescribing trends for common gout medications.

Methods. An ecological study was performed using UK National Health Service (NHS) Digital Hospital Episode Statistics data to calculate the incidence of unplanned admissions with primary diagnoses of gout or RA in adults in England between April 2006 and March 2017. NHS Digital Community Prescription data for allopurinol, febuxostat, and colchicine were considered over a similar period.

Results. The incidence of unplanned gout admissions increased by $58.4 \%$ over the study period, from 7.9 admissions per 100,000 population in $2006 / 07$ to 12.5 admissions per 100,000 population in $2016 / 17$ ( $p<0.0001$ ). Gout admissions increased as a proportion of all hospital admissions, and accounted for 349,768 bed-days cumulatively. Unplanned RA admissions halved over the study period, from 8.6 admissions per 100,000 population in $2006 / 07$ to 4.3 admissions per 100,000 population in 2016/17 ( $<<0.0001$ ). Community prescriptions dispensed for allopurinol and colchicine have increased by $71.4 \%$ and $165.6 \%$, respectively, since 2006 ( $<<0.0001)$. Febuxostat prescriptions have increased 20 -fold since 2010 ( $\mathrm{p}<0.0001$ ), when prescription data became available.

Conclusion. Acute gout admissions in England increased between 2006 and 2017, accompanied by increasing prescription of gout therapies. Acute admissions due to RA halved over the same time period. These data call for aggressive target-driven therapy for this highly treatable disease. (First Release February 15 2020; J Rheumatol 2020;47:619-23; doi:10.3899/jrheum.190257)
\end{abstract}

Key Indexing Terms:

GOUT RHEUMATOID ARTHRITIS EPIDEMIOLOGY HEALTH SERVICES RESEARCH

Gout is the most common inflammatory arthritis, with a rising incidence and prevalence worldwide ${ }^{1,2,3,4,5,6}$. Between 1997 and 2012, the incidence of gout in the United Kingdom increased by $29.6 \%$, and the prevalence increased by $63.9 \%{ }^{1}$. Gout is recognized as having deleterious effects on the musculoskeletal system, including irreversible erosive arthritis, and has a strong association with cardiovascular and

From the Centre for Rheumatic Diseases, King's College London, London; Nottingham University Hospitals UK National Health Service (NHS)

Trust, Nottingham; Division of Rheumatology, Orthopaedics and Dermatology, University of Nottingham, Nottingham, UK.

M.D. Russell, MB BChir, MA, MRCP, Centre for Rheumatic Diseases, King's College London; $M$. Yates, $M B C h B, B S c, M R C P$, Centre for Rheumatic Diseases, King's College London; K. Bechman, MBChB, BSc, MRCP, Centre for Rheumatic Diseases, King's College London;

A.I. Rutherford, MBBS, MSc, MRCP, Centre for Rheumatic Diseases, King's College London; S. Subesinghe, BSc, MBBS, MSc, MRCP, Centre for Rheumatic Diseases, King's College London; P. Lanyon, DM, MRCP, Nottingham University Hospitals NHS Trust, and Division of

Rheumatology, Orthopaedics and Dermatology, University of Nottingham; J.B. Galloway, MBChB, MSc, MRCP, PhD, Centre for Rheumatic Diseases, King's College London.

Address correspondence to Dr. J.B. Galloway, Centre for Rheumatic Diseases, NIHR Biomedical Research Centre at Guy's and St. Thomas NHS Foundation Trust and King's College, Weston Education Centre, King's College Hospital, Denmark Hill, London SE5 9RS, UK.

E-mail: james.galloway@kcl.ac.uk

Accepted for publication August 23, 2019. renal comorbidities ${ }^{7,8,9,10}$. There are highly effective, low-cost treatments available for acute gout flares [nonsteroidal antiinflammatory drugs (NSAID), colchicine, and corticosteroids] and flare prevention [urate-lowering therapy (ULT) such as allopurinol and febuxostat]. Timely commencement of ULT with appropriate dose up-titration to target serum urate levels limits further crystal formation and helps to eliminate preexisting crystals, resulting in fewer gout attacks, prevention of longterm joint damage, and a lower risk of renal function decline ${ }^{10}$. However, previous studies have shown that gout is suboptimally managed; in 2012, only $27.3 \%$ of incident gout patients received ULT within 12 months of diagnosis, and only $39.7 \%$ of patients receiving ULT were adherent to treatment ${ }^{1}$. There have since been concerted efforts to raise awareness about gout management and encourage prescription of ULT, with recently updated management guidelines from the British Society for Rheumatology (BSR) and European League against Rheumatism (EULAR) ${ }^{10,11}$.

All healthcare systems are trying to improve their productivity and efficiency, with a focus on avoidable admissions. We examined the inpatient burden of gout by describing trends in admissions to secondary care for gout in England between 2006 and 2017, using rheumatoid arthritis (RA) as a comparator. We also examined prescribing trends in

Personal non-commercial use only. The Journal of Rheumatology Copyright (c) 2020. All rights reserved. 
England for 3 common gout medications (allopurinol, colchicine, and febuxostat) over a similar time period.

\section{MATERIALS AND METHODS}

Data sources. A descriptive ecological study was performed using 2 population-level datasets to ascertain admission and prescription trends. Hospital admissions data were obtained from the Hospital Admitted Patient Care Activity records, which form part of the Hospital Episode Statistics (HES) dataset ${ }^{12}$. The HES dataset includes coded data on primary and secondary diagnoses for all admissions to UK National Health Service (NHS) hospitals in England from April 1 to March 31 of each year. Prescription data were obtained from the Prescription Cost Analysis datase $^{13}$, a record of all prescriptions dispensed in the community in England from January 1 to December 31 of each year. Both datasets are made publicly available on a yearly basis by NHS Digital in their aggregate form. Only aggregate, anonymized data were used in our analyses; no patient-level data were used, and in accordance with national guidance, no ethical approval was required. Data validation of primary events was not possible, and there were no means of accessing or accounting for missing data. Mid-year population estimates for England were obtained from the Office for National Statistics ${ }^{14}$

Study period and study population. The study periods for admission and prescription data were April 1, 2006, to March 31, 2017, and January 1, 2006 , to December 31,2016, respectively; the difference was due to the time frames included in the respective datasets. The focus of our admission analyses was trends in unplanned admissions, rather than elective admissions such as day-case attendances for joint aspiration and/or injection. Analyses included only admissions of persons aged 20 years and older, chosen because the vast majority (99.94\%) of gout admissions fell within this age range.

Incidence of gout and RA admissions. For each year of admission data (April 1 to March 31) in the study time period, the incidence of unplanned gout admissions in adults in England was calculated and displayed as an annual incidence per 100,000 population, the numerator being the number of unplanned finished admission episodes with primary diagnoses of gout, and the denominator being the mid-year population estimate for people aged 20 and above in England. A finished admission episode was defined as the first period of inpatient care under 1 consultant within 1 healthcare provider. As a comparator, the incidence of unplanned admissions with primary diagnoses of RA in adults was calculated using the same methodology.

Length of stay and cumulative bed-day consumption. For gout admissions, we provided annual figures for mean and median lengths of stay in hospital, obtained from the HES dataset, and described how the length of stay has changed over the study period. We described how the annual number of finished consultant episode bed-days attributable to admissions where gout was the primary diagnosis changed between 2006 and 2017, and calculated the cumulative number of bed-days attributable to gout admissions over this time period. Planned and unplanned gout admissions were included in these analyses because of the characteristics of the data included in the aggregate HES dataset.

Diagnostic coding inclusion and exclusions. Diagnoses in the HES dataset are coded according to the International Classification of Diseases, 10th revision. For gout, the vast majority of admissions were coded as "gout, unspecified" (M10.9) or "idiopathic gout" (M10.0). A small number of admissions due to secondary causes of gout were also included in our analyses: lead-induced gout (M10.1), drug-induced gout (M10.2), gout due to impairment of renal function (M10.3), and other secondary gout (M10.4). Pseudogout admissions were not included in our analyses. Excluded diagnostic codes were hydroxyapatite deposition disease (M11.0), familial chondrocalcinosis (M11.1), other chondrocalcinosis (M11.2), other specified crystal arthropathies (M11.8), and crystal arthropathy, unspecified (M11.9).

For RA, the majority of admissions were coded as "rheumatoid arthritis, unspecified" (M06.9), "seropositive rheumatoid arthritis, unspecified" (M05.9) or "seronegative rheumatoid arthritis" (M06.0). Additional diagnostic codes included were rheumatoid lung disease (J99.0, M05.1), Felty's syndrome (M05.0), rheumatoid vasculitis (M05.2), rheumatoid arthritis with involvement of other organs and systems (M05.3), other seropositive rheumatoid arthritis (M05.8), rheumatoid bursitis (M06.2), rheumatoid nodule (M06.3), other specified rheumatoid arthritis (M06.8), and palindromic rheumatism (M12.3). Excluded diagnoses were adult-onset Still disease (M06.1), inflammatory polyarthropathy (M06.4), and juvenile rheumatoid arthritis (M08.0)

Prescription trend analyses. Three medications (allopurinol, colchicine, and febuxostat) were selected for prescription trend analyses owing to their frequent use and relative specificity for the treatment of gout. Allopurinol and colchicine had prescription data available for the full study period. Febuxostat received UK National Institute for Health and Care Excellence approval in 2008, and had prescription data available from 2010 onward. All preparations of allopurinol, colchicine, and febuxostat listed in the Prescription Cost Analysis dataset were included: allopurinol tablets (100 mg, $300 \mathrm{mg})$, allopurinol liquid $(10 \mathrm{mg} / 5 \mathrm{ml}, 150 \mathrm{mg} / 5 \mathrm{ml}$, $15 \mathrm{mg} / 5 \mathrm{ml}, 200 \mathrm{mg} / 5 \mathrm{ml}, 20 \mathrm{mg} / 5 \mathrm{ml}, 50 \mathrm{mg} / 5 \mathrm{ml}, 5 \mathrm{mg} / 5 \mathrm{ml})$, allopurinol oral solution $(100 \mathrm{mg} / 5 \mathrm{ml}, 100 \mathrm{mg} / 5 \mathrm{ml}$ sugar free, $300 \mathrm{mg} / 5 \mathrm{ml})$, allopurinol oral suspension $(100 \mathrm{mg} / 5 \mathrm{ml}, 300 \mathrm{mg} / 5 \mathrm{ml})$; Caplenal tablets (100 mg, $300 \mathrm{mg})$, Cosuric tablets $(100 \mathrm{mg})$, Uricto tablets $(100 \mathrm{mg}$, $300 \mathrm{mg})$, Xanthomax-100 tablets (100 mg), Xanthomax-300 tablets (300 mg), Zyloric tablets (100 mg), Zyloric-300 tablets (300 mg), colchicine oral solution $(250 \mathrm{mcg} / 5 \mathrm{ml})$, colchicine tablets $(250 \mu \mathrm{g}, 500 \mu \mathrm{g})$; febuxostat tablets $\left(80 \mathrm{mg}, 120 \mathrm{mg}\right.$ ), and Adenuric tablets $(80 \mathrm{mg}, 120 \mathrm{mg})^{13}$. NSAID and corticosteroids were not selected for analysis because of the multitude of treatment indications in addition to gout. We also excluded several less frequently used gout treatments owing to the small numbers, or lack of, community prescriptions: benzbromarone, probenecid, sulfinpyrazone, rasburicase, pegloticase, and interleukin 1 inhibitors.

For each year of prescription data in the study period, we adjusted the number of dispensed prescriptions for each medication for the mid-year population estimate of adults in England, displayed as the number of dispensed prescriptions per 100,000 population.

Statistical analysis. Chi-square tests for trend were performed to assess the statistical significance of trends identified in the following over the study period: annual incidences of gout and RA admissions; mean and median lengths of stay for gout admissions; annual bed-day consumption for gout admissions; and medication prescriptions. Trends were deemed to be statistically significant at $\mathrm{p}<0.05$. All statistical analyses were performed using Stata 14.0 (StataCorp.).

\section{RESULTS}

All-cause admissions. Between April 2006 and March 2017, there was an annual average of 14.9 million all-cause hospital admissions in England, 35.4\% of which were unplanned admissions. All-cause admissions (planned and unplanned) increased by $27.5 \%$ over the study period, from 13.0 million admissions in 2006/07 to 16.5 million admissions in 2016/17; unplanned admissions increased by $25.2 \%$, from 4.7 million in 2006/07 to 5.9 million in 2016/17. When adjusted for the mid-year population estimates for England, there was a 15.4\% increase in the incidence of unplanned all-cause admissions, from 9222 admissions per 100,000 population in $2006 / 07$ to 10,645 admissions per 100,000 population in 2016/17.

Gout admissions. Over the study period, there was an annual average of 5148 admissions in which gout was the primary admission diagnosis in adults, $82.0 \%$ of which were unplanned admissions. The incidence of unplanned gout admissions in adults increased by $58.4 \%$ over the study 
period, from 7.9 admissions per 100,000 population in $2006 / 07$ to 12.5 admissions per 100,000 population in 2016/17 (Figure 1). This increase was statistically significant using a chi-square test for trend ( $<<0.0001)$, and was well above the $15.4 \%$ background increase in the incidence of all-cause unplanned admissions. In contrast, unplanned admissions with primary diagnoses of RA (which represented $4.2 \%$ of all primary RA admissions, the vast majority being elective/day-case admissions) decreased by $50.2 \%$ over the study period, from 8.6 admissions per 100,000 population in $2006 / 07$ to 4.3 admissions per 100,000 population in $2016 / 17$ $(\mathrm{p}<0.0001 ;$ Figure 1).

Unplanned gout admissions increased as a proportion of all unplanned hospital admissions, from $0.065 \%$ in 2006/07 to $0.090 \%$ in $2016 / 17$, while unplanned RA admissions decreased as a proportion of all unplanned admissions, from $0.071 \%$ in $2006 / 07$ to $0.031 \%$ in $2016 / 17$.

Length of stay. The mean length of stay for gout admissions (planned and unplanned) was 7 days in 2006/07 and 6 days in 2016/17; the median length of stay was 4 days in 2006/07 and 3 days in 2016/17. The changes in mean and median lengths of stay for gout admissions were not statistically significant $(\mathrm{p}=0.40$ and $\mathrm{p}=0.55$, respectively). In comparison, the mean length of stay for all-cause hospital admissions was 6 days in 2006/07 and 5 days in 2016/17; the median length of stay was 2 days in 2006/07 and 1 day in 2016/17. Cumulatively, gout admissions accounted for 349,768 bed-days between April 2006 and March 2017, increasing from 24,804 attributable bed-days in $2006 / 07$ to 34,389 attributable bed-days in 2016/17 ( $<0.0001$ ).

Prescription data. Community prescriptions dispensed in England for allopurinol, colchicine, and febuxostat between January 2006 and December 2016 are shown in Table 1. Prescriptions for allopurinol increased by $71.4 \%$ over the study period, from 7412 prescriptions per 100,000 population in 2006 to 12,707 prescriptions per 100,000 population in 2016 ( $\mathrm{p}<0.0001$ ). Colchicine prescriptions increased by $165.6 \%$, from 394 prescriptions per 100,000 population in 2006 to 1046 prescriptions per 100,000 population in 2016 $(\mathrm{p}<0.0001)$. Febuxostat prescription data were available from 2010. Since then, prescriptions for febuxostat have increased nearly 20 -fold, from 15 prescriptions per 100,000 population in 2010 to 300 prescriptions per 100,000 population in $2016(\mathrm{p}<0.0001)$.

\section{DISCUSSION}

We found that unplanned gout admissions in England increased by 58.4\% between April 2006 and March 2017, while unplanned RA admissions halved over the same time period. Gout admissions have increased as a proportion of all hospital admissions; accounting for 349,768 bed-days, cumulatively, over the study period. Community prescriptions for 3 common gout medications (allopurinol, colchicine, and febuxostat) increased by $71.4 \%, 165.6 \%$, and 20 -fold, respectively.

Our findings complement those of previous studies, which

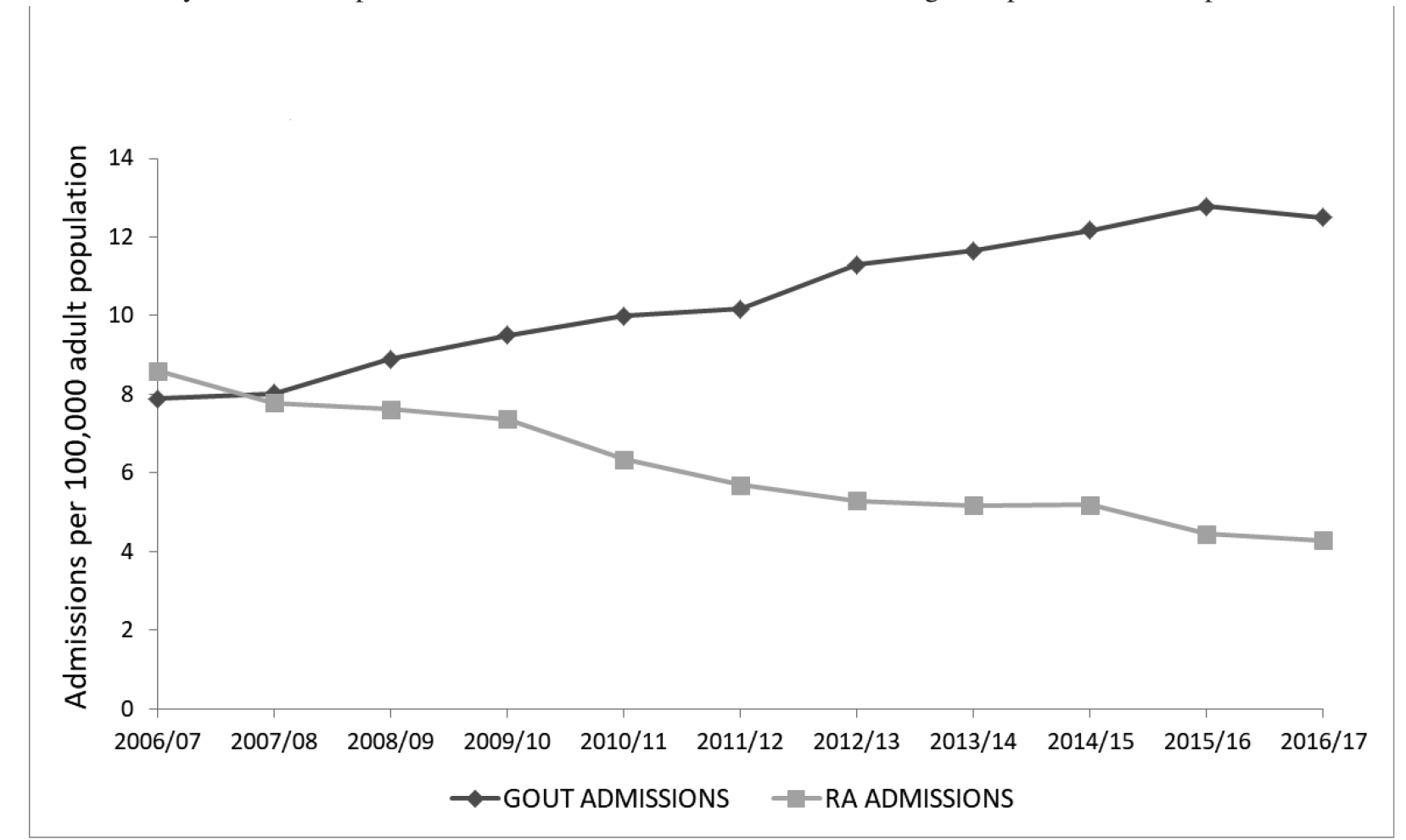

Figure 1. Trends in the incidence of unplanned admissions due to gout and RA in England between April 2006 and March 2017. Incidences are given per 100,000 adult population. RA: rheumatoid arthritis.

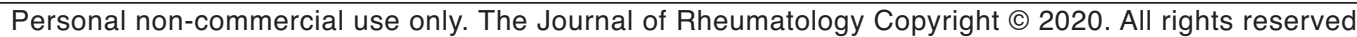


Table 1. Community prescriptions dispensed in England for allopurinol, colchicine, and febuxostat between January 2006 and December 2016.

\begin{tabular}{|c|c|c|c|c|c|c|}
\hline \multirow[t]{2}{*}{ Year } & \multicolumn{2}{|c|}{ Allopurinol } & \multicolumn{2}{|c|}{ Colchicine } & \multicolumn{2}{|c|}{ Febuxostat } \\
\hline & No. Prescriptions & $\begin{array}{l}\text { Prescriptions per } \\
100,000 \text { Population }\end{array}$ & No. Prescriptions & $\begin{array}{l}\text { Prescriptions per } \\
100,000 \text { Population }\end{array}$ & No. Prescriptions & $\begin{array}{l}\text { Prescriptions per } \\
100,000 \text { Population }\end{array}$ \\
\hline 2006 & $2,856,557$ & 7412 & 151,783 & 394 & - & - \\
\hline 2007 & $3,042,428$ & 7822 & 168,058 & 432 & - & - \\
\hline 2008 & $3,260,541$ & 8302 & 197,628 & 503 & - & - \\
\hline 2009 & $3,470,461$ & 8766 & 226,606 & 572 & - & - \\
\hline 2010 & $3,691,230$ & 9234 & 250,192 & 626 & 5982 & 15 \\
\hline 2011 & $3,911,632$ & 9683 & 286,883 & 710 & 23,638 & 59 \\
\hline 2012 & $4,200,148$ & 10,314 & 327,503 & 804 & 41,350 & 102 \\
\hline 2013 & $4,515,803$ & 11,005 & 373,555 & 910 & 63,739 & 155 \\
\hline 2014 & $4,850,422$ & 11,713 & 406,721 & 982 & 86,664 & 209 \\
\hline 2015 & $5,111,110$ & 12,233 & 424,863 & 1017 & 104,647 & 250 \\
\hline 2016 & $5,357,400$ & 12,707 & 441,071 & 1046 & 126,284 & 300 \\
\hline
\end{tabular}

have shown an increasing inpatient burden of gout in many countries worldwide $2,3,4,5,15,16$. A study of hospitalization trends in the United States using the Nationwide Inpatient Sample database demonstrated an increase in the annual hospitalization rate for patients with a primary discharge diagnosis of gout, from 4.4 to 8.8 per 100,000 adults between 1993 and 2011; this contrasted with RA, where a reduction from 13.9 to 4.6 hospitalizations per 100,000 adults was seen ${ }^{16}$. Hospitalizations due to gout in Canada doubled between 2000 and 2011, while hospitalizations due to RA fell by $49 \% 2$. A registry study performed in Sweden between 1998 and 2015 also highlighted a doubling of gout hospitalizations, contrasting with a $78.9 \%$ reduction in RA hospitalizations ${ }^{4}$. Further, our data demonstrate that the increase in gout admissions evident in England between 1999 and $2009^{15}$ has continued inexorably in recent years.

A key driving force behind the increasing number of gout admissions demonstrated in our study is likely to be the rising incidence and prevalence of gout in the UK general population ${ }^{1}$. This has been associated with an ageing, multimorbid population, changing lifestyle and dietary factors, and a growing epidemic of the metabolic syndrome ${ }^{17}$. Management of gout also remains suboptimal; fewer than a third of incident gout patients received ULT within 12 months of diagnosis in a large UK-based primary care cohort in $2012^{1}$. In those prescribed ULT, adherence was poor ${ }^{1}$. Although community prescriptions for gout medications were seen to increase in our study, we are unable to comment on adherence or whether appropriate dose escalation occurred to meet serum uric acid targets. Recently published BSR national audit data on gout management in UK rheumatology clinics found that serum uric acid targets of $\leq 360 \mu \mathrm{mol} / 1$ and $<300 \mu \mathrm{mol} / \mathrm{l}$ were achieved in only $45 \%$ and $25 \%$ of patients, respectively, by 12 months ${ }^{18}$. An audit of gout management in UK primary care noted similar findings, with only $38 \%$ of ULT-treated patients with gout reaching a target serum uric acid level of $\leq 360 \mu \mathrm{mol} / 1{ }^{19}$.

A significant advantage of this study is the use of population-level datasets, rather than sampled data, providing us with reliable estimates of gout admissions across England. These datasets also enable us to collect quantifiable data on the economic burden of gout, including length of stay and bed-day consumption data. The mean and median lengths of stay for gout admissions remained relatively long in 2016/17, at 6 and 3 days, respectively, with no significant changes occurring during the study period. Prolonged hospital stays, on a background of increasing numbers of gout admissions, place further strain on health resources: the NHS England 2018/19 National Tariff for a single gout admission varied from $£ 852$ for up to a 5-day admission in a patient with minimal comorbidities, to $£ 5662$ for an admission lasting up to 53 days in a patient with maximal comorbidities ${ }^{20}$.

An important limitation with descriptive analyses is the inability to make inferences about the directionality of identified trends. For example, it is likely that the observed increase in the number of prescriptions for common gout medications has occurred in response to the growing burden of gout in hospitals and the general population. We cannot exclude the possibility that the increasing number of prescriptions for ULT may have contributed to the increase in gout admissions due to inadequate co-prescription of prophylaxis. The concomitant increase in colchicine prescriptions over the study period would go against this; however, it is not possible to differentiate colchicine prescriptions for acute gout flares versus longer-term prophylaxis in these aggregate datasets. Population-level studies are also subject to the ecological fallacy, whereby making inferences about individuals based on aggregate data can be misleading. Analyses of individuallevel data is required to delineate whether the observed increase in gout admissions has been predominantly due to first presentations to hospital, re-admissions, or a combination of each.

Historically, there have been concerns about the accuracy of the diagnostic coding upon which the HES dataset is based and its potential effect on the interpretation of admission trends. The introduction of the Payment by Results payment

Personal non-commercial use only. The Journal of Rheumatology Copyright @ 2020 . All rights reserved 
system into NHS Trusts between 2003/04 and 2006/07 was associated with significant improvements in the accuracy of diagnostic coding, particularly the primary diagnostic coding upon which this study is based ${ }^{21,22}$. We can therefore be more assured about the accuracy of diagnostic coding data included in our study time frame. Further, our demonstration that gout admissions have increased as a proportion of all hospital admissions, while RA admissions have fallen over the study period, suggests that the observed rise in gout admissions was not down to improvements in diagnostic coding alone. One must also consider admissions in which gout flares occurred as secondary diagnoses, rather than primary diagnoses; these data will not have been recorded in our analyses, leading to an underestimation of the overall inpatient burden of gout.

Our finding of an increase in gout admissions since 2006 must act as a call-to-arms to rheumatologists and front-line clinicians alike to ensure we aggressively manage this condition. We need to seize the opportunity to reduce the 34,389 bed-days per annum currently being used for the inpatient treatment of gout attacks in England. Potential strategies include the introduction of a "door-to-needle" time to encourage the prompt aspiration of joint effusions to help elucidate the diagnosis, alleviate symptoms, and ultimately, reduce the length of stay. Timely access to admission avoidance pathways, for example, urgent-access rheumatology clinics and specialist nurse helplines for patients with gout attacks, are likely to reduce the need for admission. These pathways have been widely adopted for other inflammatory arthritides, particularly RA, and are likely to have contributed to the decline in the number of acute hospital admissions due to RA. Most importantly, adoption of recent guidelines recommending that ULT be offered to all gout patients, combined with education about the importance of adherence, dose escalation, and flare prophylaxis will be essential if successes in the management of RA are to be replicated with gout ${ }^{10}$.

Unplanned gout admissions increased by $58.4 \%$ in England between 2006 and 2017. There are highly effective medications available to treat and prevent gout attacks. We must seize the opportunity to reduce the inpatient and community burden of gout through more widespread use of ULT, with dose escalation to meet serum urate level targets, and increased use of admission avoidance pathways.

\section{ACKNOWLEDGMENT}

This work has been presented as an oral conference abstract at the British Society for Rheumatology Annual Conference $2018^{23}$.

\section{REFERENCES}

1. Kuo C, Grainge MJ, Mallen C, Zhang W, Doherty M. Rising burden of gout in the UK but continuing suboptimal management: a nationwide population study. Ann Rheum Dis 2015;74:661-7.

2. Rai SK, Aviña-Zubieta JA, McCormick N, De Vera MA, Lacaille D, Sayre EC, et al. Trends in gout and rheumatoid arthritis hospitalizations in Canada from 2000 to 2011. Arthritis Care Res
2017;69:758-62.

3. Elfishawi MM, Zleik N, Kvrgic Z, Michet CJ Jr, Crowson CS, Matteson EL, et al. The rising incidence of gout and the increasing burden of comorbidities: a population-based study over 20 years. J Rheumatol 2018;45:574-9.

4. Kiadaliri AA, Englund M. Temporal trends and regional disparity in rheumatoid arthritis and gout hospitalizations in Sweden, 1998-2015. Clin Rheumatol 2018;37:825-30.

5. Robinson PC, Kempe S, Tebbutt I, Roberts L. Epidemiology of inpatient gout in Australia and New Zealand: temporal trends, comorbidities and gout flare site. Int J Rheum Dis 2017;20:779-84.

6. Kuo CF, Grainge MJ, Zhang W, Doherty M. Global epidemiology of gout: prevalence, incidence and risk factors. Nat Rev Rheumatol 2015;11:649-62.

7. Kuo CF, See LC, Luo SF, Ko YS, Lin YS, Hwang JS, et al. Gout: an independent risk factor for all-cause and cardiovascular mortality. Rheumatology 2010;49:141-6.

8. Roddy E, Mallen CD, Doherty M. Gout. BMJ 2013;347:f5648.

9. Stamp LK, Chapman PT. Gout and its comorbidities: implications for therapy. Rheumatology 2013;53:33-44.

10. Hui M, Carr A, Cameron S, Davenport G, Doherty M, Forrester H, et al; British Society for Rheumatology Standards, Audit and Guidelines Working Group. The British Society for Rheumatology Guideline for the Management of Gout. Rheumatology 2017;56:1056-9.

11. Richette P, Doherty M, Pascual E, Barskova V, Becce F, Castañeda-Sanabria J, et al. 2016 updated EULAR evidence-based recommendations for the management of gout. Ann Rheum Dis 2017;76:29-42.

12. NHS Digital. Hospital admitted patient care activity. [Internet. Accessed January 13, 2020.] Available from: digital.nhs.uk/ data-and-information/publications/statistical/ hospital-admitted-patient-care-activity

13. NHS Digital. Prescription cost analysis. [Internet. Accessed January 13, 2020.] Available from: digital.nhs.uk/data-and-information/ publications/statistical/prescription-cost-analysis

14. Office for National Statistics. Population estimates. [Internet. Accessed January 13, 2020.] Available from: www.ons.gov.uk/peoplepopulationandcommunity/ populationandmigration/populationestimates

15. Robinson PC, Merriman TR, Herbison P, Highton J. Hospital admissions associated with gout and their comorbidities in New Zealand and England 1999-2009. Rheumatology 2013;52:118-26.

16. Lim SY, Lu N, Oza A, Fisher M, Rai SK, Menendez ME, et al. Trends in gout and rheumatoid arthritis hospitalizations in the United States, 1993-2011. JAMA 2016;315:2345-7.

17. Doherty M. New insights into the epidemiology of gout. Rheumatology 2009;48 Suppl 2:ii2-8.

18. Roddy E, Packham J, Obrenovic K, Rivett A, Ledingham JM. Management of gout by UK rheumatologists: a British Society for Rheumatology national audit. Rheumatology 2018;57:826-30.

19. Cottrell E, Crabtree V, Edwards JJ, Roddy E. Improvement in the management of gout is vital and overdue: an audit from a UK primary care medical practice. BMC Fam Pract 2013;14:170.

20. NHS Improvement. National tariff payment system $2017 / 18$ and 2018/19. [Internet. Accessed January 13, 2020.] Available from: improvement.nhs.uk/resources/national-tariff-1719

21. Burns EM, Rigby E, Mamidanna R, Bottle A, Aylin P, Ziprin P, et al. Systematic review of discharge coding accuracy. J Public Health 2012;34:138-48.

22. Herbert A, Wijlaars L, Zylbersztejn A, Cromwell D, Hardelid P. Data resource profile: hospital episode statistics admitted patient care (HES APC). Int J Epidemiol 2017;46:1093-i.

23. Russell MD, Yates M, Rutherford AI, Subesinghe S, Bechman K, Lanyon P, et al. Return of the king: rising incidence of gout in England 2006-2017 (abstract). Rheumatology 2018;57 Suppl 3:004.

Personal non-commercial use only. The Journal of Rheumatology Copyright (C 2020. All rights reserved. 